\title{
Pensar y re-pensar la producción académica en el campo de las artes:
}

\section{una reflexión a partir de las experiencias docentes en una universidad al sur de Chile*}

THINK AND RE-THINK THE ACADEMIC PRODUCTION IN THE FIELD OF ARTS: REFLECTIONS FROM TEACHING EXPERIENCES IN A UNIVERSITY

IN THE SOUTH OF CHILE

PENSAR E REPENSAR A PRODUČ̃O ACADÊMICA NO CAMPO DAS ARTES: UMA REFLEXÃO SOBRE A EXPERIÊNCIAS EDUCACIONAIS DE UMA UNIVERSIDADE SUL DO CHILE

\section{Ignacio Soto Silva** \\ Paola Alvarado Toledo*** Jorge Ferrada Sullivan****}

Cuadernos de Música, Artes Visuales y Artes Escénicas

/ Volumen 12 - Número 1 / Enero - Junio de 2017

/ ISSN 1794-6670/ Bogotá, D.C., Colombia / pp. 133-142

Fecha de recepción: 18 de noviembre de 2015

Fecha de aceptación: 8 de junio de 2016

Disponible en línea: 20 de febrero de 2017

doi:10.11144/Javeriana.mavae12-1.prpa

* Ensayo

** Doctorando en Musicología EN la Universidad de Valladolid (España). Es miembro del colectivo escena sur Peuma y del Consejo General de la Red Interuniversitaria de Educación e Interculturalidad (RIEdl). Actualmente es académico del Departamento de Humanidades y Artes y de la Carrera de Pedagogía en Educación Media en Artes de la Universidad de los Lagos.

*** Magíster en Ciencias de la Educación, intérprete y creadora. Integra el Colectivo Escena sur Peuma y es jefa de la Carrera de Pedagogía en Educación Media en Artes de la Universidad de Los Lagos.

**** Doctor en Filosofía y magíster en Artes. Profesor de Historia y Geografía en la Universidad de Chile. Actualmente es académico del Departamento de Humanidades y Artes y de la Carrera de Pedagogía en Educación Media en Artes de la Universidad de los Lagos. 


\section{Resumen}

El presente ensayo surge con el objetivo de reflexionar sobre la base de la integración de los campos de investigación científica y el arte, sus prácticas y estrategias metodológicas como medio de unificación de criterios de calidad en una universidad al sur de Chile. Dicho proceso surge a propósito de la situación actual de la universidad, las dimensiones del desarrollo artístico y su relación con la academia, las cuales en el contexto mencionado se encuentran tensionadas por la productividad científica y sus cánones de valoración. Estas premisas valóricas se contraponen (en el aquí y ahora) a la lógica de funcionamiento de la creación artística desarrollada en las universidades periféricas. Esta reflexión se estructura a partir del posicionamiento epistemológico de Morin y Bourdieu. Asimismo, según los planteamientos teóricos de otros autores que buscan proponer esquemas metodológicos que contribuyan a la validación de la actividad científica y artístico-creativa en la academia.

Finalmente, se busca propiciar la interacción reflexiva de posturas teóricas que permitan plantear en las diversas instancias del desarrollo académico actual una relación dialogante entre el saber sensible del arte, la productividad científica y sus implicancias en los procesos de valoración académica en docentes de carreras de pregrado asociadas al arte.

Palabras claves: investigación artística; creación artística; producción académica; saberes situados.

\section{Abstract}

The production of knowledge in the University professor of arts is always product of the interaction between the art and the other disciplines of sciences. In this regard we understand that the professor in academic context has to make own the dynamics of production of sciences. Furthermore, we know that the actual situation in Chilean universities and the contemporary framework of the art production in the academy are in tension with the canon of scientific research.

Finally, we don't want to answer the questions emerged in this article, in fact we want to make contrast with diverse theoretical purposes with the aim of encourage the interaction of diverse forms of knowledge, scientific and sensible. Also, we want to re-think the implications of these forms of knowledge in the process of valuation of academic performance.

Keywords: artistic research; artistic creation; academic production; situated knowledge.

\section{Resumo}

Começamos a nossa reflexão sobre a base da integração dos campos da investigação científica e arte, suas práticas e estratégias metodológicas como um meio de critérios de qualidade unificadora. Também entendo que, na situação actual da Universidade, as dimensões do desenvolvimento artístico e sua relação com a academia são tensionados pela produtividade científica e padrões de avaliação, que se opõem -no aqui e agora- a lógica operação da criação artística desenvolvida no contexto das universidades periféricas

Finalmente, não se destina a responder às perguntas, pelo contrário, destina-se a discutir posições teóricas que permitem propor uma relação de diálogo entre o conhecimento sensível da arte, a produtividade científica, e suas implicações no processo de avaliação acadêmica em ensino de graduação associado à Art.

Palavras chave: pesquisa artística; criação artística; produção acadêmica; conhecimento localizado. 


\section{OBERTURA}

El presente artículo emerge desde las reflexiones conjuntas de los autores, llevadas a cabo a propósito del seminario Epistemologías y ciencias en el sur: narrativas subyacentes de la producción de conocimiento en la universidad, realizado en la Universidad de Los Lagos (Osorno, Chile) durante los días 18 y 19 de junio de 2015. El objetivo principal es por tanto analizar las prácticas artísticas y sus implicancias en los procesos de jerarquización y validación en el marco universitario, desde un posicionamiento situado, es decir, tomando en consideración la realidad territorial que nos convoca (la zona sur-austral de Chile).

Comprendemos, en este sentido, que la productividad artística actual en la "universidad periférica" —entendida como la universidad de provincia, con escaza interacción con los centros de producción artística masivos, localizados en sectores centralizados- no satisface los criterios de valoración necesarios para equipararse a la investigación científica, por cuanto hasta el momento no podemos validar, por ejemplo, un concierto coral de fin de año con una ponencia en congreso, o aún más, una performance en un espacio público con una publicación indexada. Esto marca un distanciamiento entre ambos campos de estudio, lo cual se evidencia en las complejidades que surgen en un artista en el momento de asumir su papel como académico en la universidad.

Expresamos aquí la necesidad de un diálogo conjunto entre los campos científico y artístico, ya que, como menciona Fajardo (2008), la universidad espera que el artista contribuya a una construcción sistemática del conocimiento, tanto en la producción como en la reflexión (87), lo cual evidentemente debe ir de la mano con su productividad artística como creaciones o interpretaciones. Como mencionábamos en el párrafo anterior, uno de los principales cuestionamientos surge desde nuestras propias prácticas, en cuanto nos preguntamos si en efecto una exposición pictórica puede llegar a valorizarse como una publicación o una ponencia en congreso. Y bajo qué condiciones o variables es posible definirlo como tal. Es un tema complejo, porque nos muestra una realidad subyacente en la producción de los artistas que se encuentran inmersos en la academia —al menos en el ámbito regional en Chile—, además de un problema no resuelto aún en la universidad desde una perspectiva genérica.

En este sentido, O'Riley (2011) menciona que tanto el arte como la ciencia comparten elementos de raíz común, sus procedimientos son semejantes en su génesis, no obstante, las diferencias son notorias en cuanto a la puesta en marcha de ambas, y el punto crítico es la valoración y evaluación del rigor en el campo artístico. Proponemos entonces un posicionamiento dual por parte del artista, es decir, este debe adoptar el papel de artista-investigador, creador-investigador o intérprete-investigador. Las formas de validar la producción científica difieren en rigor de manera evidente con la producción artística, por tanto se hace necesario cuestionarnos cómo el artista-académico puede adoptar los códigos de divulgación científica e incorporarlos en su lenguaje y práctica. En definitiva, concluimos que este debe empoderarse de su papel como académico universitario, desde un conocimiento y saber sensible.

La estructura de esta reflexión se concibe en una primera instancia mediante la discusión respecto de las tensiones existentes entre el campo de la ciencia y arte, sus diálogos y el posicionamiento actual. Junto con esto, y como menciona Bergdorff (2010), se analiza la noción de investigación artística, concepto que conecta los dominios — del arte y la ciencia—, por cuanto permite al artista racionalizar sus procesos creativos. Esta discusión es basal, porque comprender cómo se configura la relación entre el arte y la ciencia nos permitirá también dilucidar 
las modalidades de generación de conocimientos que emergen desde ellas. En este sentido, los planteamientos de Chiantore (2014) y los propuestos por los autores de esta comunicación nos llevan a preguntarnos ¿qué clase de conocimiento persigue la investigación artística? ¿No es el arte (y su obra) en sí mismo el producto de una investigación? ¿La consagración del artista en cuanto docente universitario está definida en la opinión y crítica de sus pares o en su productividad académica? Esto nos remite necesariamente al diálogo de tales campos como eje central en la labor del profesor universitario y sitúa el estudio del arte desde el arte al centrarse en los procesos creativos y en la racionalización de estos como fuente para la generación de conocimientos que contribuyen a la depuración de los aspectos estéticos y técnicos del artista. Zaldivar (2008) define a la investigación desde el arte como

la "cara oculta" del estudio sobre el arte, pues no se centra en el objeto artístico, ni en el documento que lo explica de una u otra manera, ni en la biografía del creador, ni en la respuesta del público o el eco en sus diversos medios de difusión y múltiples interpretaciones. La investigación "desde el arte" se centra en el propio proceso de creación. (2-3)

Nos posicionaremos en la noción de investigación artística como un proceso engendrado desde el arte. Comprendemos que las perspectivas paradigmáticas en cuanto a la investigación en artes nacen desde dos aristas epistémicas: desde el arte y sobre el arte. El posicionamiento investigativo sobre el arte nos remite a una práctica antigua en el marco universitario, muy valiosa y ampliamente realizada en el ámbito nacional (no así en el local, es decir, suraustral). En este sentido, las perspectivas que tratan el estudio del arte como objeto de investigación se configuran desde un enfoque multidisciplinar que en estricto rigor viene a adoptar estrategias metodológicas propias de las diversas ciencias humanas y sociales.

\section{CIENCIA Y ARTE: TENSIONES EPISTEMOLÓGICAS EN RELACIÓN CON LOS CAMPOS DE PRODUCCIÓN}

La producción científica y la creación artística han sido siempre dimensiones distanciadas en cuanto a los soportes epistemológicos y ontológicos que definen estos campos de producción. Por una tradición histórica, lo científico, y su carácter intelectual metodológico, ha sido disociado del habitus del creador y de la reflexión que podrían poseer las obras y los procesos creativos en cuanto una mirada investigativa que el mismo artista —entendido en este caso como artista-académico universitario - realiza del fenómeno. En términos concretos, inferimos la existencia de una serie de supuestos intervinientes en dicha reflexividad: interpelaciones o preguntas "científicas" por la obra, el complejo entre-tejido artístico del proyecto como objeto de intencionalidad pensante y quizá la formación escolar y universitaria —carente de prácticas inteligibles- que reciben los iniciados en el campo artístico. Más bien ha sido el carácter estético en dicho proceso lo que ha definido y primado en diversos intelectuales y teóricos a lo largo de las épocas. Sin duda alguna, la tendencia de dicha producción en las últimas décadas se ha visto complementada por el aporte de filósofos y escritores contemporáneos, como Michel Foucault, Jacques Derrida, Jean-Luc Nancy, Pierre Bourdieu, Edgar Morin, entre otros, que han puesto en evidencia el espesor de la tensión en aquellas producciones culturales y en la visualidad de los factores intervinientes en la constitución de los campos científico-intelectual y el arte. Sus interrogaciones han presentado cuestiones como aquellas referidas a procesos de legitimación, validación y proyección del trabajo intelectual y artístico 
con todas las implicancias y complejidades que poseen bajo suposiciones en torno al proceso artístico como foco de investigación y tensión metodológica. Preguntas como ¿quién crea a los intelectuales en su posicionamiento?, ¿quién crea a los creadores en su validación social?, ¿existirá una relación de jerarquías entre el campo científico-intelectual y el artístico?, ¿es desde la universidad el origen de las distancias epistemológicas de la relación arte y ciencia? En otras palabras, ¿el fenómeno artístico puede ser investigado por los propios artistas desde la academia? o ¿un artista y su obra serán reconocidos culturalmente por la producción académica en su nombre? Algo cierto hay en estas cuestiones: toda la literatura relacionada con lo artístico, con los procesos creativos, con la obra de arte y sus fundamentos, ha sido narrada por intelectuales que precisamente no son artistas y que desde lo académico-universitario han legitimado a los creadores bajo la supeditación del alero científico en desmedro de la capacidad reflexiva que puede poseer el artista. Parafraseando a Morin (1982), no es de extrañar, entonces, que bajo miradas tradicionales del desarrollo científico el principio de explicación de la ciencia clásica tendiera a reducir lo conocible a lo manipulable, "habría que insistir con fuerza en la utilidad de un conocimiento que pueda servir para ser reflexionado, meditado, discutido, incorporado por cada uno en su saber, su experiencia, su vida (46)".

Como una premisa inicial, se puede sostener que tanto los productores científico-intelectual y artistas-creadores no se relacionan de modo directo con el mundo social, sino a través de la estructura de un corpus intelectual —originado desde la universidad y para la investigación académica - que ha actuado como mediador entre la ciencia social, el arte y la cultura. Sin embargo, como sostiene Juanola (1997), "el propio arte es un campo propicio de estudio para estos propósitos; si en la obra se objetivan las ideas, si el artista concreta interpretaciones esenciales de las cosas, surge inmediatamente la cuestión de las relaciones entre arte y ciencia" (4). En efecto, la relación que un creador sostiene con su obra está entre otras condicionantes directamente relacionada con la escritura crítica en el campo intelectual, ya que es este quien configura la definición y la evolución del proyecto creador. Sin embargo, esta cuestión plantea ciertas tensiones conflictivas en el momento de preguntarse: ¿la consagración del artista está definida en la opinión y crítica del intelectual desde la investigación universitaria? Pierre Bourdieu (2003) sostiene que el artista puede aceptar o repudiar al intelectual que la sociedad le envía, pero no puede ignorarlo: el artista debe enfrentar la definición social de su obra, es decir, concretamente, los éxitos o fracasos que esta tiene, las interpretaciones que de ella se dan, la representación social, a menudo estereotipada y reductora, que de ella se hace el público de los aficionados. Así es como se puede sostener que el sujeto de la obra corresponde a una relación entre el habitus y su posición en campo productivo académico. Desde ese lugar es donde se produce la particular determinación con el público, el cual tiene que procesar que el sujeto-creador de la producción artística y de su obra no es solo el artista, sino el conjunto de fuerzas que participan en el campo del arte. Siguiendo esta idea

se debe tomar por objeto no solamente las condiciones sociales de la producción de los productores (es decir, los determinantes sociales de la formación o de la selección de los artistas), sino también las condiciones sociales de producción del campo de producción como lugar donde se realiza el trabajo que tiende a (y no que pretende) producir al artista como productor de objetos sagrados. (Bourdieu 1999, 217) 
Desde la universidad la producción científico-intelectual toma en cuenta la legitimidad de su escritura a partir de la diversidad de formas de divulgación (catálogos, capítulos de libros, artículos en revistas, ponencias), que entran en el círculo social y se convierten en una suerte de capital legitimado por la investigación académica. Para Bourdieu, "el dominio del imperio de la economía sobre la investigación artística o científica también se ejerce en los campos, a través del control de los medios de producción y de difusión cultural, e incluso, de las instancias de consagración" (2003, 192).

La relación con el capital social en la universidad moderna es también fuerte, pues la producción académica se hace parte de la sociedad bajo sus estrategias de validación no solo como grupo de intelectuales e investigadores sobre el arte -en la que ocupan una posición-, sino también en todas las relaciones sociales y políticas que los involucra. Se puede interpretar que a mayor acumulación y autonomía del capital en el campo académico —en cuanto científico-intelectual o artístico- mayor es el grado de legitimación que posee el productor en los círculos universitarios e intelectuales. Es el caso de los centros de investigación, sociedades académicas universitarias, círculos disciplinares o grupos pequeños en las disciplinas, instancias de publicación o transmisión —como revistas disciplinares- del saber de dominación legítimo.

Considerando que el intelectual —académico bajo su exposición científica y el artista y obra en circulación - se ha constituido y creado en estos campos de producción, articulados por todos quienes han contribuido a descubrirlos, conocerlos y reconocerlos en círculos científicos y de crítica cultural, no es menor la consolidación de estrategias de resguardo y validación, que introducen a los intelectuales y artistas a círculos de participación académica y de valoraciones de bienes artísticos y simbólicos. Demás está decir que el circuito de consagración está determinado por la instalación de la literatura del intelectual y del artista en universidades, líneas editoriales de interés científico, exposiciones y publicaciones en catálogos. Pero insistimos en la problemática de este texto: los artistas no han sido reconocidos en su autonomía como investigadores de sí mismos o sobre sus procesos creativos, sino desde las instancias universitarias que resguardan la supremacía del hábito científico por sobre el artístico.

\section{GENERAR CONOCIMIENTO DESDE EL ARTE: EL ARTISTA COMO INVESTIGADOR DE SÍ MISMO}

La noción de investigar desde el arte implica necesariamente fijar la mirada desde el artista como investigador de su propio proceso creativo y centrara el interés en el cómo él mismo produce o recrea la obra de arte. Esta reflexión permite que el artista pueda aprender de su propia labor y del trabajo de los otros; en consecuencia, si los profesores y estudiantes de artes en el contexto universitario investigan sobre sus propias formas de generar procesos creativos o recrear obras de arte, pueden eventualmente depurar sus prácticas creadoras e interpretativas, y con ello mejorar —en su dimensión de profesor universitario- de forma eficiente la docencia de la práctica artístico-creadora, en vistas de contribuir a la formación de un artista intelectual.

Zaldívar (2006) denomina a este tipo de investigación "creativa performativa", por cuanto esta se relaciona con la idea de acción artística y a su vez con el posicionamiento de Judith Butler —es decir, actuar desde el discurso— y romper así con el prejuicio de lo intuitivo o inexplicable del 
arte. En este sentido, se busca quebrar el tabú de la actividad práctica artística, en su dimensión creativa o "performativa" y su idealización como misterio poético, anticientífico, romántico e inefable, en definitiva contraria a la noción de investigación científica (Zaldivar 2006, 7).

De acuerdo con lo anterior, es imprescindible encontrar vías adecuadas para que tanto la creación y la "recreación artística" —entendida en este punto como la puesta en escena de una obra de teatro, danza o música ya estrenada- puedan ser críticamente estudiadas y visibilizar sus procesos y proponer así conclusiones valorables para la comunidad científica por cuanto de estas emerge conocimiento sensible. Esto es un reto que en el campo artístico nos llama a la apertura de nuevos horizontes, es decir, la pesquisa desde el arte, desde el artista como investigador de sí mismo, lo cual puede colaborar a complementar y enriquecer las perspectivas epistémicas tanto "sobre el arte" como en otros campos del conocimiento.

Para desarrollar investigación sobre el proceso creativo propio del artista, podemos tomar como referente a Bruner (1991), citado por Hernández (2008), para quien el conocimiento y la creación humana se divide en una modalidad "paradigmática", que busca la experiencia basándose en la prueba lógica, el análisis razonado, la observación empírica y la narrativa, centrada en el ser humano, en sus intenciones, experiencias, deseos y necesidades. En complemento, para Bruner son la narrativa biográfica y el relato de vida modalidades que operan de una manera similar a una conversación entre el modo pragmático y el modo narrativo/imaginativo (89). En este sentido, y desde el campo metodológico, podemos aludir a la autoetnografía como herramienta metodológica validadora en los procesos de autoindagación del quehacer creativo. López-Cano y San Cristóbal $(2014,22)$ ponen énfasis en las metodologías autoetnográficas (Heider 1975), las cuales, si bien son mencionadas en gran cantidad de trabajos, no han sido definidas ni explicadas — en el campo de la investigación artística - a fondo. La aproximación metodológica autoetnográfica es para Ellis, Adams y Bochner $(2011,273)$ un concepto referido a una serie de estrategias que tienen como objeto describir y analizar la experiencia personal del investigador en busca de comprender aspectos elementales de los fenómenos en los cuales se encuentra inmerso. En esta línea, López-Cano y San Cristóbal (2014) mencionan que la información autoetnográfica está centrada en el sujeto mismo, y en su interacción con otras personas, así como en su interacción con objetos materiales o culturales (140).

Desde el campo de la investigación artística, el uso de estrategias metodológicas autoetnográficas nos posibilita sistematizar eventos desarrollados durante el proceso mismo de la investigación-creación, ya sea en su pasado, ya sea presente, y permite su autonomía como investigador de sí mismo (López-Cano y San Cristóbal 2014). Cabe destacar esto como una diferencia fundamental respecto de la acepción de la autoetnografía desde la antropología. Así es como en la investigación artística el concepto de autoetnográfica no considera al investigador exclusivamente como representante de una cultura o fenómeno del que este forma parte, sino que se enfoca en él mismo (142) y en sus procesos creativos.

En cuanto a las bases para llevar a cabo procesos de investigación desde el arte, encontramos los planteamientos de Zaldívar (2008, 11-12), quien lo expresa en los siguientes puntos:

- Como toda ciencia, busca la transparencia en el proceso y necesita asegurar la comunicabilidad de los resultados.

- XXIII Salón de Artistas Nacionales (Museo Nacional, Bogotá, 1972) 
- Es necesariamente inter-subjetiva, pero de modo riguroso y crítico (nunca arbitraria, vanidosa ni engañosa).

- Narra la experiencia, le interesa sobre todo el proceso creativo (sin despreciar el resultado).

- Es esencial poseer un elevado nivel de experiencia y conocimientos de la creación o interpretación.

- Es posible considerarla científica gracias a la crisis de la investigación "clásica" —que exigía una "objetividad" hoy insostenible incluso para las ciencias experimentales-y en gran medida es posible su realización y transmisión más efectivas debido a las modernas tecnologías.

- El resultado de esta investigación ha de servir para crear e interpretar mejor, para el propio artista o para quienes se comunican sus resultados.

Habla y transmite con rigor lo que en la idealización del artista romántico era/es lo "inefable" (aunque se pueden considerar interesantes precedentes, quitándoles el almíbar decimonónico, ciertas interesantes propuestas románticas que nos relatan procesos creativos e interpretativos).

- Ha de delimitar progresivamente sus temas y metodologías.

- No es igual a investigación histórico-artística ni filosófico-estética, ni es musicología o teoría de la literatura, etc. (aunque muchas de las habilidades y saberes de todas esas ciencias les sean directa o indirectamente precisos).

En complemento a lo planteado por Zaldivar, López-Cano y San Cristóbal (2015) definen también el perfil del artista investigador, quien necesariamente debe: 1. Generar reflexión continúa sobre su propia práctica artística. 2. Problematizar aspectos de su actividad artística y de su entorno con la finalidad de ofrecer diagnósticos, análisis, reflexiones y soluciones. 3. Construir un discurso propio respecto de su propuesta artística y hacer énfasis en argumentación sólida sobre su aporte para el arte. 4. Dejar atrás la zona de confort para ingresar en un ámbito lleno de interrogantes e incertidumbres donde el artista investigador se pone del revés constante y finalmente. 5. Integrarse en una espiral de producción y discusión de conocimiento que, como toda empresa de investigación, terminará por transformar el statu quo, es decir, las prácticas artísticas hegemónicas (81-82).

\section{REFLEXIONES FINALES}

Los artistas e intelectuales se inclinan cada vez con mayor frecuencia a la necesidad de divulgar comentarios especializados que contribuyen en forma directa a la producción científica o artística a través de su relato y reflexión. Queda de manifiesto cuando Bourdieu define estas nuevas condiciones, particularmente aquellas referidas a la producción de arte:

La constitución de un conjunto sin precedente de instituciones de registro, de conservación y de análisis de las obras (reproducciones, catálogos, revistas de arte, museos que abren sus puertas a las obras más recientes, etc.), el incremento del personal dedicado, total o parcialmente, a la solemnización de la obra de arte, a la intensificación de los intercambios de las obras y de los artistas, con las grandes exposiciones internacionales y la proliferación de galerías con varias sucursales en distintos países, etc. El discurso sobre la obra de arte no es un mero aditivo, destinado a favorecer su aprehensión y su valoración, sino un momento de la producción de la obra de arte, de su sentido y de su valor. (Bourdieu 2003: 259) 
En consecuencia, para comprender las relaciones de producción entre las fuerzas científico-intelectuales y artísticas es necesario ubicarlas —-tanto para académicos y artistas como para su exposición - dentro de una red de relaciones que se establecen entre los agentes que están en contacto directo con la producción y el mundo universitario, en su formación disciplinar reflexiva. Este sistema de relaciones no solo comprende al intelectual, al artista como académico y al público receptor, sino también a los otros científicos, intelectuales, profesores, escritores y artistas, quienes desde su posicionamiento investigativo "sobre el arte" determinan las condiciones específicas de producción y circulación de los productos académicos y artísticos. La importancia de esta cuestión radica en que el proceso de validación y legitimación de un intelectual o de un artista se articula necesariamente en estas condiciones que determinan el nombre o re-nombre en el círculo social y universitario, igual que sus códigos de producción y divulgación. La resultante es que este sistema de relaciones de legitimación académica y de creadores tendrá que tener en cuenta no solo a los productores directos de escrituras científicas y de las obras en su materialidad (artistas), sino también al conjunto de los individuos y de las instituciones que participan en la producción del valor de legitimación de artículos publicables y de creaciones de arte que pueden actuar sobre los circuitos académicos y artísticos, sea mediante veredictos de consagración acompañados o no de ventajas económicas (subvenciones, premios, becas, etc.), sea mediante medidas reglamentarias (ventajas fiscales concedidas a los productores y creadores), sin olvidar a los miembros de las instituciones que concurren a la producción de los productores (universidades, centros de investigación, escuelas de artes, conservatorios) y a la producción de públicos aptos para reconocer la escritura científica y la obra de arte como tal.

Entonces el perfil del académico que emerge desde esta reflexión, como artista-investigador, implica comenzar a re-pensar la forma de verse a sí mismo y adoptar una actitud reflexiva respecto de su quehacer e incorporar los códigos de divulgación y producción de las ciencias, lo cual en fin localiza contextualmente y legitima su conocimiento sensible dentro de la universidad. Como menciona Zaldivar (2006),

de nada sirve seguir aspirando, como algunos intérpretes ingenuos -o perezososproponen, a ser considerados investigadores, y alcanzar el doctorado a partir de una mera acumulación de conciertos triunfales. Tampoco el cirujano, titulado superior indiscutible [...] alcanza su condición oficial de investigador, ni obtiene el grado de doctor, por mera suma de operaciones de éxito. (7)

La investigación desde el arte nos lleva a deconstruir los discursos y desmitificar las posturas esencialistas del arte como fenómeno, de una actividad inspirada e imposible de transmitir o criticar, hacia un proceso dinámico, reflexivo y generador de realidades situadas. Finalmente, las modalidades de generación de conocimiento en la universidad necesariamente deben responder a las realidades de las disciplinas, de igual manera las disciplinas deben tributar a las formas de divulgar y transmitir saberes de la academia. Entendemos esto como un llamado de atención a las prácticas actuales de los artistas creadores e intérpretes, por cuanto su divorcio con la praxis académica tensiona y dificulta los procesos de validación de su trabajo en un entorno universitario. Asimismo comprendemos el desafío que propone este cambio paradigmático, puesto que las problemáticas expuestas no son un fenómeno exclusivo a la realidad de esta universidad, sino que son un problema recurrente en los centros académicos regionales, por tanto, un fenómeno nacional. 


\section{REFERENCIAS}

Borgdorff, Henk. 2010. "The Production of Knowledge in Artistic Research". En The Routledge Companion to Research in the Arts, editado por Henrik Karlsson y Michael Biggs. Londres: Routledge.

Bourdieu, Pierre. 1999. Intelectuales, política y poder. Buenos Aires: Eudeba.

- 2003. Cuestiones de sociología. Madrid: Istmo.

Bruner, Jerome. 1991. Actos de significado: la autobiografía del yo. Madrid: Alianza.

Chiantore, Luca. 2014. "Prólogo." En Investigación artística en música: problemas, métodos, experiencias y modelos, editado por Rubén López-Cano y Úrsula San Cristóbal. Barcelona: Programa de Fomento a Proyectos y Coinversiones Culturales del Fondo Nacional Para la Cultura y las Artes de México.

Declaración y recomendaciones del Congreso Internacional Qué Universidad para el mañana? Hacia una evolución transdisciplinar de la Universidad. 1997. Locarno, Suiza, abril-mayo, $30-2$.

Ellis, Carolyn, Tony Adams y Arthur Bochner. 2011. "Autoethnography: An Overview". Historical Social Research/Historische Sozialforschung 12: 273-290.

Fajardo, Roberto. 2008. "La investigación en el campo de las artes visuales en el ámbito académico universitario". Revista de Ciencias Sociales y Humanísticas Universidad de Panamá 10: 85-98.

Heider, Karl. 1975. "What Do People Do? Dani Auto-Ethnography." Journal of Anthropological Research 31: 3-17.

Hernández, Fernando. 2008. "La investigación basada en las artes: propuestas para repensar la investigación en educación." Educación Siglo XXI 26: 85-118.

Juanola iTerradellas, Roser. 1997. "Arte, ciencia y creatividad: un estudio de la escuela operativa italiana". Arte, Individuo y Sociedad 9: 11-32.

López-Cano, Rubén y Úrsula San Cristóbal. 2014. Investigación artística en música: problemas, métodos, experiencias y modelos. Barcelona: Programa de Fomento a Proyectos y Coinversiones Culturales del Fondo Nacional Para la Cultura y las Artes de México.

— 2015. "El dilema de la investigación artística". Anais do SIMPOM 3 (3). http://seer.unirio.br/ index.php/simpom/article/viewFile/4482/4055

Morin, Edgard. 1982. Ciencia con conciencia. México: Multidiversidad-Mundo Real.

O'Riley, Tim. 2011. A Discrete Continuity: On the Relation Between Research and Art Practice. Journal of Research Practice, 7 (1). http://jrp.icaap.org/index.php/jrp/article/viewArticle/257

Zaldívar Gracia, Álvaro. 2006. "El reto de la investigación creativa y performativa." Eufonía 38: 87-94.

- 2008. "Investigar desde el arte". Anales de la Real Academia Canaria de Bellas Artes de San Miguel Arcángel 1: 57-6.

\section{Cómo citar este artículo:}

Soto Silva, Ignacio; Alvarado Toledo, Paola \& Ferrada Sullivan, Jorge. 2017. "Pensar y re-pensar la producción académica en el campo de las artes: una reflexión a partir de las experiencias docents en una universidad al sur de Chile" Cuadernos de Música, Artes Visuales y Artes Escénicas 12 (1): 133-142. doi:10.11144/Javeriana.mavae12-1.prpa. 